\title{
Subtalar dislocation: rare and often forgotten
}

\author{
Uei Pua
}

Received: 20 October 2008 / Accepted: 7 January 2009 / Published online: 14 February 2009

(C) Springer-Verlag London Ltd 2009

A 17-year-old boy presented to the emergency department (ED) with pain and ankle deformity following an inversion injury during a basketball game. Radiographs of the right ankle (Fig. 1) showed loss of talonavicular alignment (white arrow) and widening of the talocalcaneal joint (black arrows). Furthermore, the midfoot was medially displaced in relation to the ankle (curved arrow). The findings are consistent with medial subtalar dislocation. Following failed manipulation and reduction $(M \& R)$ in the $E D$, successful closed M\&R was achieved under general anaesthesia.

Medial subtalar dislocation is a rare dislocation and is not commonly seen as a sports injury as it requires transfer of a large amount of kinetic energy [1]. The weaker talocalcaneal and talonavicular ligaments often bear the brunt of the energy and are more commonly disrupted, compared to the relatively stronger calcaneonavicular ligament. Due to the high energy nature, up to $40 \%$ of

U. Pua $(\bowtie)$

Department of Diagnostic Radiology, Tan Tock Seng Hospital,

11 Jalan Tan Tock Seng,

Singapore 308433, Singapore

e-mail: druei@yahoo.com dislocations are associated with open injury or have significant internal soft tissue injury related to ligament disruption. Clinically, it is important to recognize the midfoot deformity, and the foot is typically supinated and held in plantar flexion. This uncommon midfoot deformity is often not appreciated and standard ankle radiographs are performed. Nevertheless, prompt M\&R prior to imaging should be attempted in all ankle injuries with significant deformity, as there is a high risk of neurovascular compromise [2]. Careful assessment and documentation of the overlying skin condition and distal neurovascular status are essential both in the acute and post-M\&R period as skin necrosis as well as neurovascular injury can occur as a result of the initial injury or the M\&R.

Ankle radiographs, if performed, appear deceptively normal as the ankle joint remains intact. Careful scrutiny of the talonavicular and talocalcaneal joints is crucial for correct diagnosis. Long-term sequelae include talar avascular necrosis [3] and osteochondral fracture [4], as well as chronic instability and pain. Urgent reduction is important, and closed reduction under general anaesthesia is usually successful, often facilitated by keeping the knee in flexion to relax the gastrocnemius muscle. However, the capsule of the talonavicular joint and the extensor digitorum brevis tendon often block reduction and open reduction will then be necessary. 
Fig. 1 Radiographs of the right ankle showed loss of talonavicular alignment (white arrow) and widening of the talocalcaneal joint (black arrows). The midfoot was medially displaced in relation to the ankle (curved arrow)

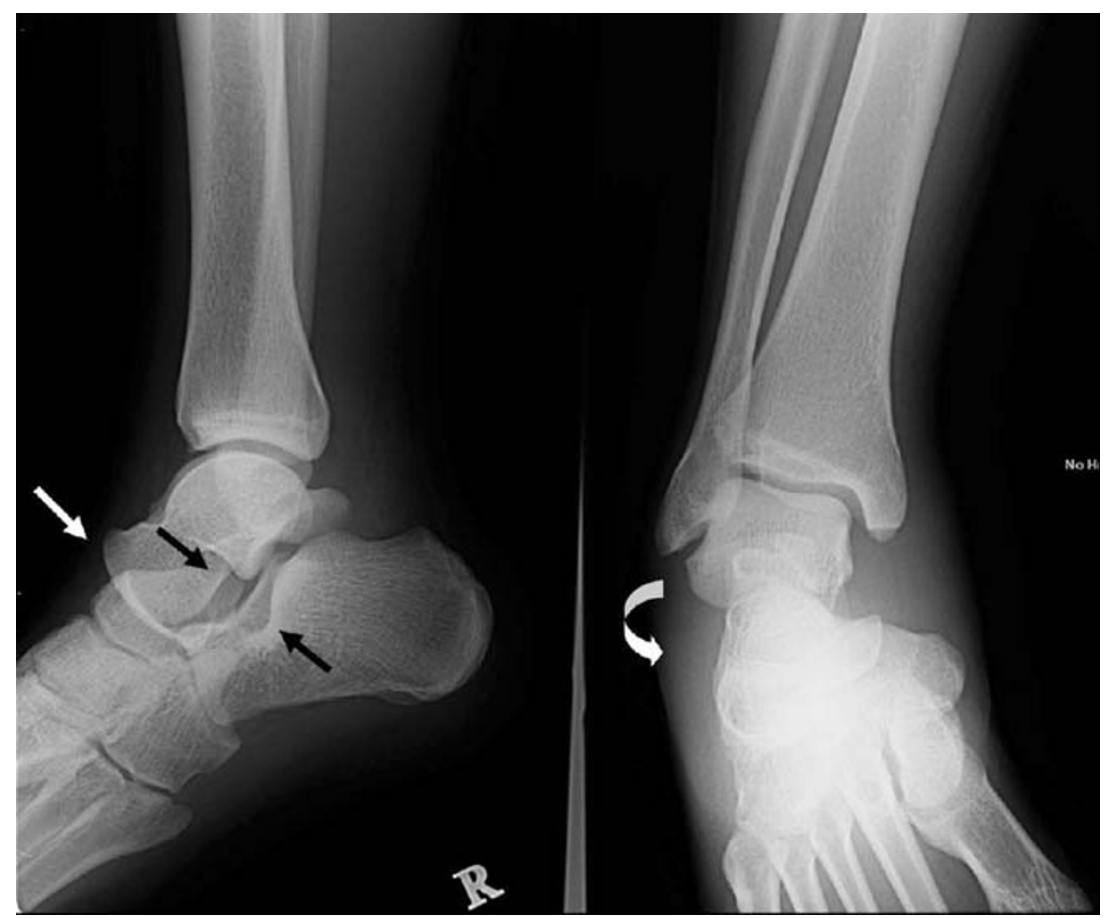

\section{References}

1. Jerome JT, Varghese M, Sankaran B (2007) Anteromedial subtalar dislocation. J Foot Ankle Surg 46(1):52-54
2. Freund KG (1989) Subtalar dislocations: a review of the literature. J Foot Ankle Surg 28(5):429-432

3. Marcinko DE, Zenker CC (1991) Peritalar dislocation without fracture. J Foot Ankle Surg 30(5):489-493

4. Bohay DR, Manoli A 2nd (1996) Occult fractures following subtalar joint injuries. Foot Ankle Int 17(3):164-169 